\title{
Topsoil radiological characterisation of L-54M reactor surroundings preliminary to Decommissioning operations
}

\author{
E. Mossini*, L. Codispoti, M. Giola, L. Castelli, E. Macerata, A. Porta, F. Campi, and M. Mariani \\ Department of Energy, Politecnico di Milano, Piazza L. da Vinci 32, I-20133 Milano \\ * Corresponding author: eros.mossini@polimi.it
}

\begin{abstract}
The radiological characterization of the topsoil of the L-54M reactor surroundings carried out in this work aims at obtaining the reference blank point for the forthcoming decommissioning operations and ascertain if unexpected radionuclide release occurred during the operational life of the plant. Standardised methods have been employed in order to collect representative samples and reliable results. Suitable sample pre-treatment procedures were applied. Gamma and beta spectrometric analyses were carried out to measure the activity concentrations of ${ }^{60} \mathrm{Co}{ }^{137} \mathrm{Cs},{ }^{152} \mathrm{Eu},{ }^{241} \mathrm{Am}$ and ${ }^{90} \mathrm{Sr}$. These have been considered as representative radionuclides that could have been originated from reactor operations and that could still be present at four decades post reactor shutdown.
\end{abstract}

Keywords: Decommissioning; Research reactor; Radiological characterization; Topsoil.

\section{Introduction}

Worldwide a number of nuclear power plants will undergo permanent shutdown and subsequent decommissioning over the next decades [1,2]. This is also the case of L-54M, a homogeneous fuel thermal research reactor $(50 \mathrm{~kW}$ nominal thermal power) commissioned by Politecnico di Milano to Atomic International in 1958 [3]. The core consists of a special steel (AISI 347) sphere of $20 \mathrm{~cm}$ radius filled up with a uranyl sulfate solution enriched by $19.94 \%$ of ${ }^{235} \mathrm{U}$. The neutron moderator and reflector of the reactor is made of nuclear grade graphite, shaped in bricks and assembled to form a parallelepiped of $7 \mathrm{~m}^{3}$ (about $11 \mathrm{t}$ ) around the core. The biological shield is made of reinforced concrete, built as a single block of $79 \mathrm{~m}^{3}$ (about $281 \mathrm{t}$ ). In order to appropriately protect the technical personnel against the neutron flux, minimum walls thickness of $170 \mathrm{~cm}$ and boron enrichment by magnetite and colemanite are used. Several neutron channels are present for materials irradiation offering a wide range of energy and intensity of the neutron flux. The position of the core is strongly asymmetric inside the monolith so as to ensure a flexible irradiation configuration. L-54M was used for research purposes in the fields of nuclear reactor physics and control, radiochemistry, radiation physic and chemistry and for materials irradiation. It was shut down in 1979 and subsequently put in Safe Storage.

After the plant shutdown, the owner of the facility was responsible for managing the end of life of the nuclear installation and defining all technical and administrative operations needed to obtain the total or partial removal of radiological restrictions imposed by the Competent Authority on the site [4]. Depending on the future business plan of the owner, the site may be reassigned to another industrial purpose where such radiological limitations are unchanged or partially changed (brownfield) or be restored to its original state and thus removing all radiological constraints (greenfield) [5]. Depending on the specific economic, political and social perspective, the management of a nuclear facility during its decommissioning is based on two main strategies: 1) Immediate 
Dismantling (DECON), when the decommissioning starts just after the plant shutdown; 2) Safe Enclosure (Deferred dismantling), when the disused nuclear power plant is locked and supervised for about 30 years and after this period the dismantling will take place. Entombment could be considered a viable solution under exceptional circumstances and envisages the permanent segregation of the nuclear power plant from the environment [6]. During the ' 80 s, some legislative gaps and the lack of a national repository resulted in the safe storage option as the only available strategy for all Italian nuclear facilities. In 2000, Italy decided to leave the waiting phase in favor of the decommissioning processes, in order to exploit the benefits that this choice entails for the Italian industry and for the radiological safety of the country. To date, preliminary activities have already been performed on the L-54M research reactor with a view of ensuring the safest storage conditions. In particular, its spent nuclear fuel (SNF) solution has already been removed from the primary circuit and sent to the Italian SNF reprocessing facility. In order to further reduce the radiological risks associated to the plant, the primary circuit has already been decontaminated and some radioactive materials and components removed. Furthermore, a pre-dismantling radiological characterization campaign aimed at estimating the radionuclides inventory has been carried out on few samples of irradiated graphite and concrete of the reactor monolith.

Among all activities, the topsoil radiological characterization of the reactor Impacted zone is one of the preliminary steps to its definitive decommissioning [6]. In the present work, radiometric analyses were systematically carried out to measure the activity concentration of selected radionuclides in topsoil samples collected in the surroundings of the L-54M reactor site. The work has been developed after a comprehensive bibliographic research, following the guidelines of the Multi-Agency Radiation Survey and Site Investigation Manual (MARSSIM) [7] and the Environmental Radiation Survey and Site Execution Manual (EURSSEM) [8]. The knowledge of the radiological inventory of the topsoil surrounding the plant defines the reference blank point that will be necessary for the final evaluation of the nuclear facility decommissioning phase. Furthermore, thanks to a comparison with the radionuclides activity concentrations measured in samples of the Non-Impacted zone, the results of the preliminary topsoil characterization are useful to estimate the impact of the reactor operative phase on the surrounding areas.

Both MARSSIM and EURSSEM provide a complete guidance on radiological, environmental and facility surveys. They are based on the best available practices for demonstrating compliance with dose or risk-based regulations of radioactively (or potentially) contaminated sites. In particular, the MARSSIM discusses thoroughly the contamination of upper layer soil and building surfaces. As initial assumption, each survey unit is considered contaminated above the release criterion until proven otherwise. The radiological surveys (scooping, characterisation) are designed to provide the information needed to reject this initial assumption. Compliance demonstration is simply a decision as to whether a survey unit meets the release criterion or not [7]. This decision is supported by statistical tests based on the results of the measurements. Depending on the type and future use of each material (any solid or dry material, not liquids or gases [9]), the Italian Regulatory Authority, ISPRA (National Institute for the Environmental Protection and Research, in Italian: Istituto Superiore per la Protezione e la Ricerca Ambientale) established Clearance Levels (CL) for the radionuclides in order to manage the disposal, recycling or re-use without restriction of the material itself. In Italy, there is not a precise regulation for assigning CL values. Using international guidelines as reference [9], the National Authority typically chooses stricter values than those recommended and, additionally, it specifies them depending on the nuclear facility under analysis, as in the case of the four SoGIN nuclear power plants and Montecuccolino nuclear reactor [10].

The main purposes of this work are: i) obtaining the reference blank point for the subsequent decommissioning activities; ii) evaluating the impact of approximately 20 years of reactor operations on the topsoil radionuclides inventory. The key radionuclides of concern when dealing with potential nuclear facility external contamination are: ${ }^{3} \mathrm{H},{ }^{14} \mathrm{C},{ }^{40} \mathrm{~K},{ }^{54} \mathrm{Mn},{ }^{55} \mathrm{Fe},{ }^{60} \mathrm{Co},{ }^{63} \mathrm{Ni},{ }^{90} \mathrm{Sr},{ }^{137} \mathrm{Cs},{ }^{152} \mathrm{Eu},{ }^{226} \mathrm{Ra},{ }^{235} \mathrm{U},{ }^{238} \mathrm{U},{ }^{232} \mathrm{Th},{ }^{241} \mathrm{Am}$ [5]. Among these, ${ }^{40} \mathrm{~K}$ 
and the progenies of ${ }^{238} \mathrm{U}$ and ${ }^{232} \mathrm{Th}$ are naturally occurring radionuclides in environmental samples. After few decades from the nuclear facility shutdown, mainly ${ }^{3} \mathrm{H},{ }^{14} \mathrm{C},{ }^{59} \mathrm{Ni},{ }^{63} \mathrm{Ni},{ }^{90} \mathrm{Sr},{ }^{137} \mathrm{Cs},{ }^{152} \mathrm{Eu}$ and the transuranium elements have to be considered accordingly with their half-lives [7]. Accordingly with the available information about L-54M reactor operations and with the absence of any accidental events, the fission products ${ }^{137} \mathrm{Cs}$ and ${ }^{90} \mathrm{Sr}$ and the Easy-to-Measure radionuclides ${ }^{60} \mathrm{Co},{ }^{152} \mathrm{Eu}$ and ${ }^{241} \mathrm{Am}$ were considered in this characterization campaign as representative markers of the environmental impact due to nuclear activities. In particular, ${ }^{90} \mathrm{Sr}$ and ${ }^{137} \mathrm{Cs}$ have been selected since they both can easily enter into biological systems and ${ }^{90} \mathrm{Sr}$, in particular, is hazardous due to its biological half-life of the order of several years [11]. Topsoil sampling and preparation were performed following International standardized protocols to guarantee representative and homogeneous samples. An aliquot of pre-treated samples was measured by gamma spectrometry to quantify ${ }^{60} \mathrm{Co},{ }^{137} \mathrm{Cs},{ }^{152} \mathrm{Eu}$ and ${ }^{241} \mathrm{Am}$, while another aliquot was subjected to a microwave-assisted digestion followed by radiochemical separation and liquid scintillation counting (LSC) of ${ }^{90} \mathrm{Y}$.

\section{Material and methods}

All reagents were used without further purification. Concentrated $\mathrm{HNO}_{3}, \mathrm{HF}$ and $\mathrm{HCl}$ were purchased from Sigma Aldrich or Alpha Aesar and used for the microwave-assisted sample digestion. A 5\% (w/v) $\mathrm{H}_{3} \mathrm{BO}_{3}$ (Merck-Millipore, $99.5 \%$ pure) solution was prepared to allow the ICP-MS analysis of the samples. The Sr-Y separations were performed with Eichrom $\mathrm{Sr}^{\circledR}$ resin, $2 \mathrm{~mL}$ prepacked cartridges, 50-100 $\mu \mathrm{m}$ grain size (Eichrom Part: SR-R50-S, Lot\# FSRS130517), mounted on Vacuum box system (Eichrom Part: AR-BOX-24), with inner support tubes (Eichrom Part: AR-1000-TUBE-PE) and yellow outer tips (Eichrom Part AR-1000-OT), connected to a vacuum pump and sealed in order ensure vacuum tightness. All solutions used for the radiochemical separation were prepared by suitable dilution of concentrated $\mathrm{HNO}_{3}$ by ultrapure water (MilliQ Merck-Millipore, USA; $18.2 \mathrm{M} \Omega \cdot \mathrm{cm}$ ). Moreover, a weighted amount of oxalic acid (Carlo Erba reagent, pure at $99.5 \%$ ) was dissolved in $3 \mathrm{M} \mathrm{HNO}_{3}$ to the final concentration of $0.05 \mathrm{M}$. A certified ${ }^{90} \mathrm{Sr}$ solution in $0.1 \mathrm{M} \mathrm{HCl}$ with specific activity of $190.00 \mathrm{~Bq} \cdot \mathrm{g}^{-1}$ (on 18/04/1986) was used to prepare the standard for the evaluation of the Cerenkov measuring efficiency. A weighted amount of $\operatorname{Sr}\left(\mathrm{NO}_{3}\right)_{2}$ salt (Carlo Erba reagent, pure at 99.5\%) was dissolved in $0.05 \mathrm{M} \mathrm{HNO}_{3}$ in order to obtain a $5 \mathrm{mg} \cdot \mathrm{mL}^{-1} \mathrm{Sr}$ carrier stock solution for the evaluation of the chromatographic separation yield.

\subsection{Sampling and sample preparation}

As regards the acquisition of the background reference, spot-like sampling was performed in the Non-Impacted zone [7], following the general criterion of spatial uniformity. The selection of the sampling points is also influenced by the historical analysis of the land in order to identify areas that were not disturbed by the years in which the reactor was in operation. Furthermore, the role of the prevailing winds in the region (from the southwest to the northeast) takes part in this selection process [12]. 5 soil samples were collected and labelled from $1 \mathrm{R}$ to $5 \mathrm{R}$, see Figure 1. 


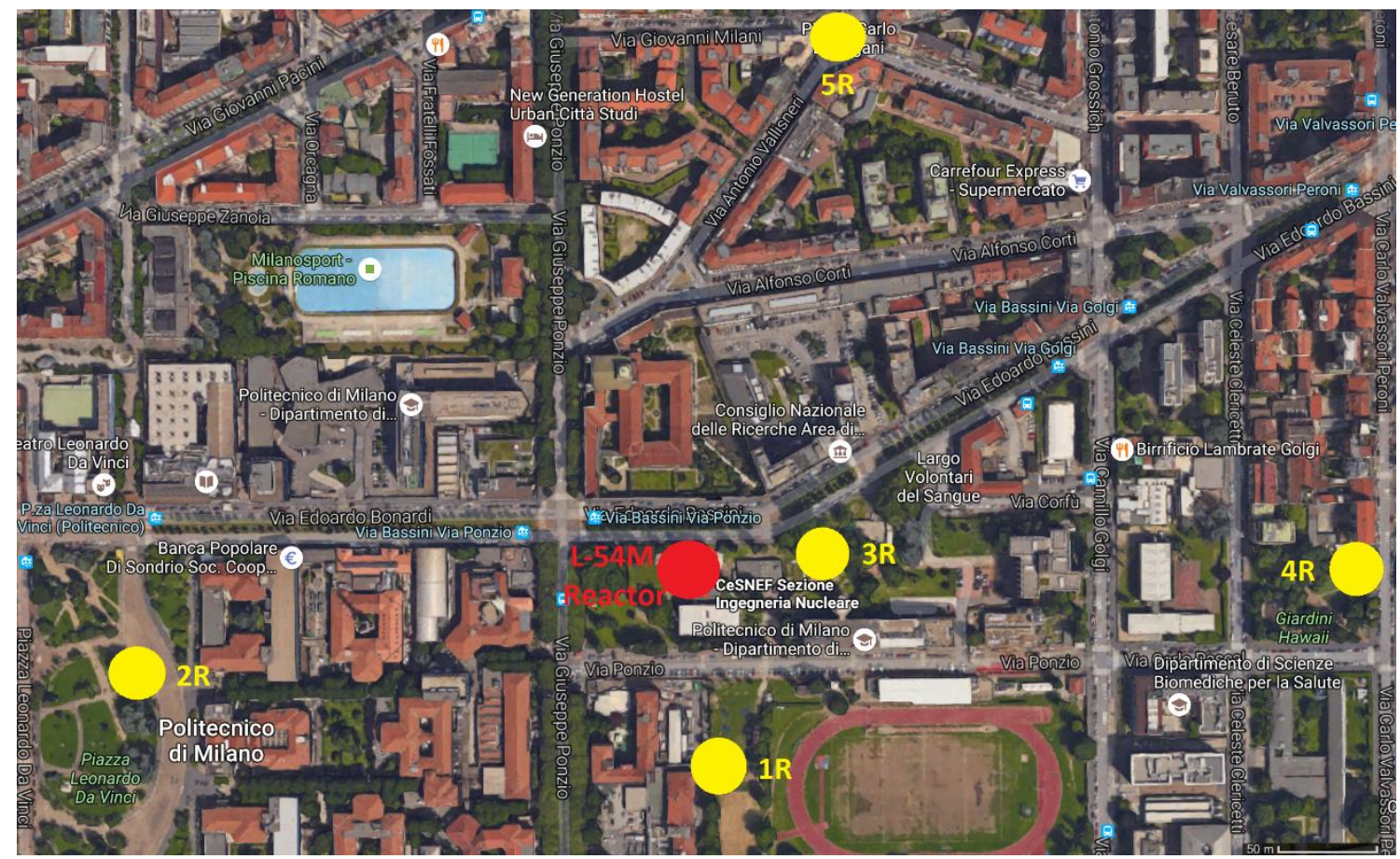

Figure 1 Map of the Non-Impacted zone sampling points.

Potentially contaminated areas due to the site operations are classified as Impacted zones. Moreover, if only a negligible contamination as compared with the background level is expected, the area is classified as Impacted zone of Class 3 [7]. According to the information collected at the beginning of the radiological characterization of the site acquired from the plant operation registry and from the experiences transmitted by the plant operators, also known as Historical Site Assessment (HSA), the whole L-54M surroundings were defined as Impacted zone of Class 3 [7]. Even if a random sampling would be advised for a Class 3 area, a more conservative systematic uniform distribution sampling was applied to this area, with grid size of about $5 \mathrm{~m}$ x $5 \mathrm{~m}$. Moreover, some sampling points were agreed according to HSA and were positioned around the reactor building (samples from 10 to 18 in Figure 2). Measuring large surfaces requires that sampling points are such that they ensure the statistical significance with a standard level of confidence that usually is set at $95 \%[7,13]$. For the minimum number of samples, the directions specified in ISO 11932 [13] regarding the clearance of recycling materials as conventional wastes are usually followed, though it is not a normative dealing with soil samples. The prescribed default number of initial samples for the entire investigation grid is 30 , but supplementary samples have to be collected if the calculated statistic criterion is not satisfied by the preliminary results. As it can be seen from Figure 2, 31 sampling points were selected. 


\section{L-54M Site - Impacted Zone of Class 3}

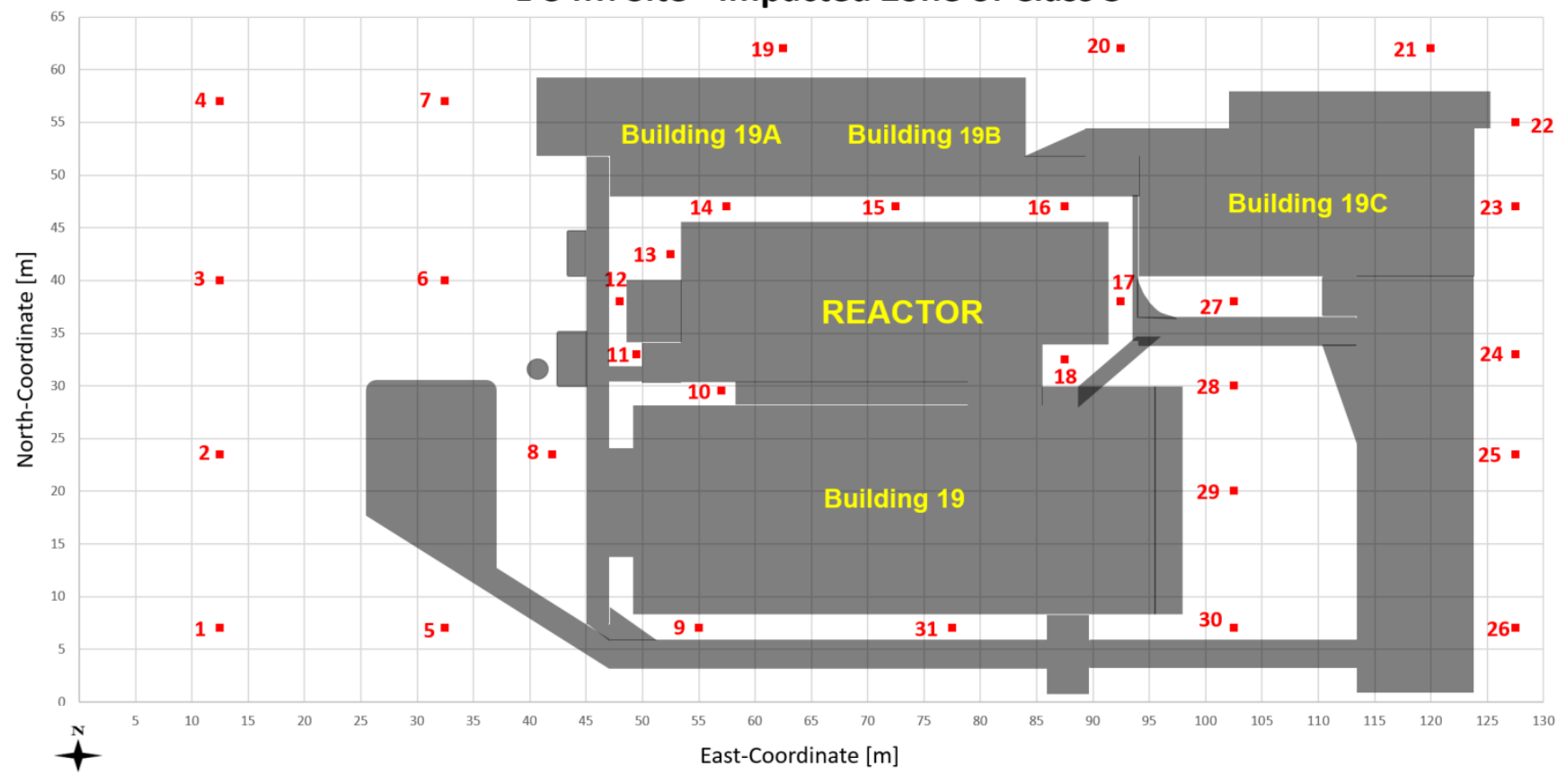

Figure 2 Map of the L-54M reactor Impacted zone sampling points.

The choice of the more suitable sampling procedure is a key aspect to be considered. Georeferenced topsoil samples were collected at the end of September 2015 following a standardised procedure [14,15]. The superficial layer of vegetation was removed and the first $2 \mathrm{~cm}$ of soil were discarded. Approximately $4 \mathrm{~kg}$ of soil, with portions of $20 \mathrm{~cm} \times 20 \mathrm{~cm}$ template and $10 \mathrm{~cm}$ depth, were picked up with a stainless steel shovel. Due to the foregone presence of humic acid in the upper layer of the soil, the interest in the sample collection was focused on the first $10 \mathrm{~cm}$. In fact, the humic acid is known to react with alkaline metal ions (such as $\mathrm{Cs}^{+}$), thus reducing their mobility and restricting their migration to the first layers of ground [16]. Topsoil samples were sieved in the field through a $20 \mathrm{~mm}$ wide mesh and then collected in transparent labelled sacks. Finally, they were weighted by a balance (10000 g capacity, $1 \mathrm{~g}$ readability). In order to avoid cross-contamination between different samples, before each operation the equipment was carefully washed with brushes and dried.

Prior to radiochemical procedures and radiometric analyses, proper soil sample manipulation in the laboratory was necessary in order to obtain a representative and homogeneous matrix for the subsequent radiometric analyses $[14,15]$. With this aim, several steps were performed on each soil portion under a radiochemical fume hood:

1. discarding vegetation, organic parts and rubble that can be present in the matrix;

2. shrinking of the moist sample mass through the so-called coning and quartering method to obtain a smaller representative matrix portion (mean weight of approximately $1 \mathrm{~kg}$ ) without introducing a systematic bias;

3. complete drying by a thermostatic oven until constant mass was reached. In order to completely remove the humidity, $24 \mathrm{~h}$ at $110^{\circ} \mathrm{C}$ turned out to be sufficient;

4. sieving with a $2 \mathrm{~mm}$ wide grid sieve to remove the matrix skeleton and to obtain a homogeneous matrix. 
In order to avoid cross-contamination during all sample manipulation, washing and covering of the working surface with a consumable plastic sheet were applied. Moreover, all equipment required an accurate washing.

\subsection{Gamma emitting radionuclides quantification}

The radiometric measures were firstly performed on the treated topsoil samples looking for a qualitative identification of the gamma emitting radionuclides $\left({ }^{60} \mathrm{Co},{ }^{137} \mathrm{Cs},{ }^{152} \mathrm{Eu}\right.$ and ${ }^{241} \mathrm{Am}$ above all $)$ and then for a quantitative determination of their activity. The apparatus consisted of a P-type coaxial High Purity Germanium (HPGe, ORTEC GEM model) detector. A lead shield (10 cm thickness) is placed around the capsule to reduce the background noise due to cosmic rays and terrestrial radiation. Inner cadmium and copper layers (1.2 mm and $1 \mathrm{~mm}$ thickness respectively), characterized by low atomic number, absorb the characteristic X-rays emitted by the bulk of the lead shield. The electronic chain consists of a pre-amplification step in the detector capsule to process the generated analogic signal. It is followed by a linear amplifier to shape the pulse to shorter width and higher amplitude, depending on the gain. An integral discriminator produces a logic output pulse only if the previous linear input pulse amplitude exceeds a discrimination level. Finally, a multichannel analyser (MCA) collects the pulse counts distribution depending on their height (in order of ascending energies), thus providing the spectrum. GammaVision MCA emulation is the software used to acquire the spectrum from the MCA, to perform the fundamental operations (e.g. calibration, background subtraction, peak identification) and to obtain the net counts associated to the $\gamma$ full energy peaks of interest, as reported in Table 1.

A fundamental parameter to calculate the activity concentration of each radionuclide in the topsoil sample is the detector absolute efficiency. It is the ratio between the recorded pulses (net counts) and the radiation effectively emitted by the source. Self-attenuation and coincidence-summing effects make the experimental estimation of this parameter more complicated so that a computational approach could be a valid aid for the evaluation of the detector efficiency. GESPECOR is a semi Monte Carlo simulation software able to physically describe the interaction between photons and matter taking into account few experimental details, such as detector and shield specifications, density and composition of the sample matrix. The software calculates the peak efficiency of the full energy peaks of the chosen library so that the efficiency calibration curve can be derived.

Table 1 Full energy peaks of the radionuclides of interest expressed in keV.

\begin{tabular}{cccc}
\hline Radionuclide & Energy [keV] & Radionuclide & Energy [keV] \\
\hline${ }^{\mathbf{6 0}} \mathbf{C o}$ & $1173.2 ; 1332.5$ & ${ }^{\mathbf{1 3 7}} \mathbf{C s}$ & 661.6 \\
${ }^{\mathbf{1 5 2}} \mathbf{E u}$ & 121.8 & ${ }^{\mathbf{2 4 1}} \mathbf{A m}$ & 59.6 \\
\hline
\end{tabular}

A sample holder characterized by cylindrical geometry was considered a good compromise between measuring efficiency and feasibility. Precisely weighted amounts of the soil samples treated as above described were transferred in $250 \mathrm{~mL}$ plastic cylindrical beakers (see Figure 3) for the gamma radiometric measurement $[14,17,18]$. In order to fill the beaker with a representative portion without introducing a systematic bias, the sample mass was shrunk through the coning and quartering method (see Figure 3). 

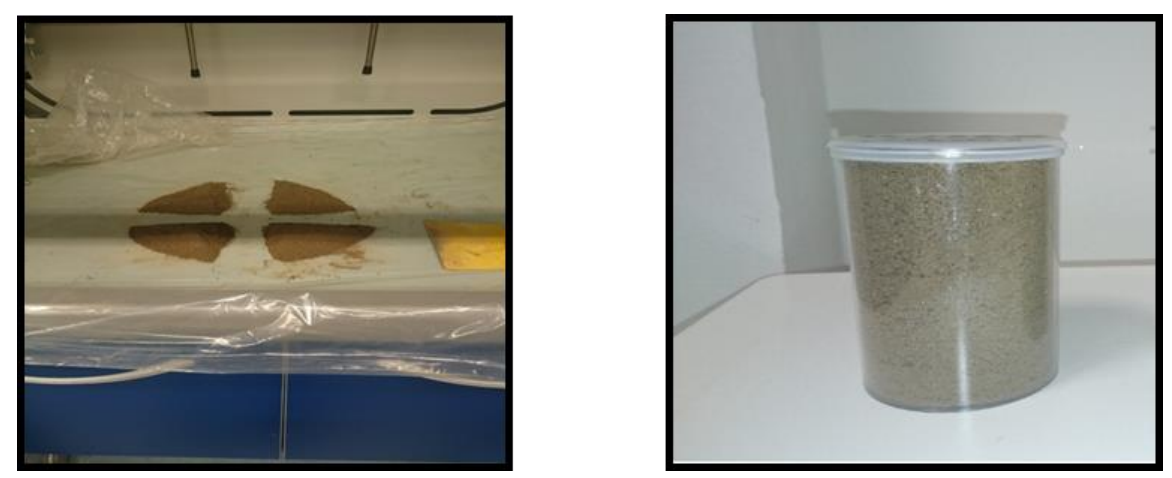

Figure 3 Coning and quartering of the dried and $2 \mathrm{~mm}$ sieved soil sample (left) to fill $250 \mathrm{~mL}$ cylindrical beaker for $\gamma$-spectrometry (right).

The measuring live time was set to $81000 \mathrm{~s}$. The same radiometric procedure was performed without filling the sample holder with the soil sample in order to acquire the blank counts associated to the measuring equipment. The activity concentration of the selected gamma emitting radionuclides was calculated according to Eq. 1 .

$$
A\left[B q \cdot g^{-1}\right]=\frac{\frac{C_{S}}{T_{S}}-\frac{c_{B}}{T_{B}}}{B R \cdot m \cdot \varepsilon}
$$

Eq. 1

where: $c_{S}$ and $c_{B}$ are the counts of the sample and of the background respectively, $T_{S}$ and $T_{B}$ are the counting live times of the sample and of the blank (81000 s and $86400 \mathrm{~s}$ respectively); $B R$ is the branching ratio; $m$ is the mass of topsoil in the cylindrical beaker; $\varepsilon$ is the detection efficiency calculated by the GESPECOR software.

\section{3. ${ }^{90} \mathrm{Sr}$ quantification}

The activity concentration of the radiological marker ${ }^{90} \mathrm{Sr}$, fission products strictly related to ${ }^{137} \mathrm{Cs}$ in the fission process, was indirectly estimated in all soil samples by measuring its progeny, ${ }^{90} \mathrm{Y}$. The measurement was carried out by liquid scintillation counting, following a focused radiochemical procedure [19,20]. A precisely weighted aliquot of the soil sample previously subjected to the gamma measurement (approximately $0.5 \mathrm{~g}$ ) was contacted with a mixture of acids and then subjected to a microwave-assisted thermal treatment in order to be digested and to recover the radionuclides from the matrix. The acid mixture consists of concentrated $\mathrm{HNO}_{3}(6$ $\mathrm{mL}), \mathrm{HF}(2 \mathrm{~mL})$ and $\mathrm{HCl}(3 \mathrm{~mL})$ according to EPA 3052 [21]. Since the artificial contaminants potentially present in the soil should not have affected the crystalline structure of the pre-existing minerals, it is worthless to completely decompose the matrix. The supernatant was separated by centrifugation from the solid residual that is discharged. In order to decompose the residual HF and allow the subsequent treatments without affecting the glass materials, $12 \mathrm{~mL}$ of $5 \%(\mathrm{w} / \mathrm{v}) \mathrm{H}_{3} \mathrm{BO}_{3}$ solution was added to the digested sample. The obtained solution was poured into a beaker and, under continuous magnetic stirring, was evaporated to dryness on a hot plate, avoiding sample leakage due to bubbles escaping from the beaker. The residues were dissolved in $10 \mathrm{~mL}$ of $8 \mathrm{M}$ nitric acid solution and were transferred in the Sr column, previously conditioned with $8 \mathrm{M} \mathrm{HNO}_{3}$, so that yttrium is released and strontium retained in the column. After the removal of the possible interfering radionuclides by $8 \mathrm{M}$ nitric acid and $0.05 \mathrm{M}$ oxalic acid in $3 \mathrm{M}$ nitric acid solutions, the strontium was eluted in a clean reservoir by 15 $\mathrm{mL}$ of $0.05 \mathrm{M}$ nitric acid to obtain the $\mathrm{Sr}$ product solution. In order to evaluate the strontium recovery yield, a precisely weighted amount of $\operatorname{Sr}\left(\mathrm{NO}_{3}\right)_{2}$ carrier solution was added before the evaporation stage and an aliquot of 
the obtained Sr product solution was analysed by ICP-MS. The Sr yield turned out to be above $90 \%$ in all the analysed samples. In order to estimate the ${ }^{90} \mathrm{Sr}$ activity concentration from its daughter measurement, the secular equilibrium between ${ }^{90} \mathrm{Sr}$ and ${ }^{90} \mathrm{Y}$ has to be guaranteed. For this reason, a waiting time of 2 weeks, correspondent to 5 times the ${ }^{90} \mathrm{Y}$ half-life, was applied. After this period, the activities of the two radionuclides are assumed to be approximately equal. A final passage in column was needed to elute ${ }^{90} \mathrm{Y}$ alone by $8 \mathrm{M}$ nitric acid. The yield of this step was assumed to be $100 \%$ according to [20]. For the radiometric measurement, the ${ }^{90} Y$ product solution has to be pure from ${ }^{40} \mathrm{~K},{ }^{214} \mathrm{~Pb}$ and other contaminants, as demonstrated by focused gamma measurements. The recovered solution was poured into a plastic vial without scintillation liquid and measured by a low background liquid scintillation counting (LSC) system Perkin Elmer Quantulus ${ }^{\mathrm{TM}} 1220$ exploiting the Cerenkov effect. In fact, beta emitting radionuclides with endpoint energy higher than the threshold energy of $263 \mathrm{keV}$, such as ${ }^{90} \mathrm{Y}$, produce detectable Cerenkov light in water or in organic solutions easily detected by a traditional liquid scintillation counting without using a scintillation cocktail [22]. The $24 \mathrm{~h}$ waiting time between the ${ }^{90} \mathrm{Y}$ recovery and the radiometric measure turned out to be adequate to avoid any undesired photo/chemi-luminescence effect. The measuring live time was set to $86400 \mathrm{~s}$. The same radiochemical procedure was performed without the soil sample in order to acquire the blank counts associated to the reagents and to the measuring equipment. Due to the inadequate correspondence between the spectrum of the continuous Cerenkov radiation and the spectral response curve of the photomultiplier, this method is known to provide scarce efficiency, around 30\% [23]. In order to experimentally assess this efficiency, a standard ${ }^{90} \mathrm{Sr}^{90} \mathrm{Y}$ solution with the two radionuclides in secular equilibrium was employed. From the counts measured by the Čerenkov $\beta$ counter, it was possible to calculate the activity concentration of ${ }^{90} \mathrm{Y}$ present in the vial, $A_{S}\left({ }^{90} Y\right)$, see Eq. 2.

$$
A_{S}\left({ }^{90} Y\right)\left[B q \cdot g^{-1}\right]=\frac{\frac{c_{S}}{T_{S}}-\frac{c_{B}}{T_{B}}}{Y_{S r} \cdot Y_{Y} \cdot I_{Y} \cdot D_{Y} \cdot \varepsilon \cdot m}
$$

where: $c_{S}$ and $c_{B}$ are the counts of the sample and of the blank respectively, $T_{S}$ and $T_{B}$ are the counting live times of the sample and of the blank (86400 s); $D_{Y}$ is the ${ }^{90} \mathrm{Y}$ decay corrective factor; $Y_{\mathrm{Y}}$ is the yttrium yield (assumed to be $100 \%) ; Y_{\mathrm{Sr}}$ is the strontium yield measured by ICP-MS; $I_{\mathrm{Y}}$ is the ${ }^{90} \mathrm{Y}$ ingrowth factor calculated as defined in [20]; $\varepsilon$ is the experimental ${ }^{90} \mathrm{Y}$ counting efficiency; $m$ is the effective mass of topsoil present in the vial. The measured $A_{S}\left({ }^{90} Y\right)$ corresponds to the activity concentration of ${ }^{90} \mathrm{Sr}$ in the soil sample. This value is referred to the dry soil sample with maximum grain size of $2 \mathrm{~mm}$.

\subsection{Detection Limit and Minimum Detectable Activity concentration}

In order to ascertain whether every single measurement can be distinguished from the background, the detection sensitivity of the measuring system was verified by comparing the sample signal with the background signal through a statistical hypothesis test. According to the statistical theory described in [24], there is a boundary value between the region of acceptance and the complementary region where the criterion is not satisfied (see Figure 4 ) that is called Critical Level ( $L_{C}$ or decision level) defined in Eq. 3.

$$
L_{C}[c p s]=\frac{1.645}{T_{S}} \cdot \sqrt{c_{B} \cdot\left(1+\frac{T_{S}}{T_{B}}\right)}
$$




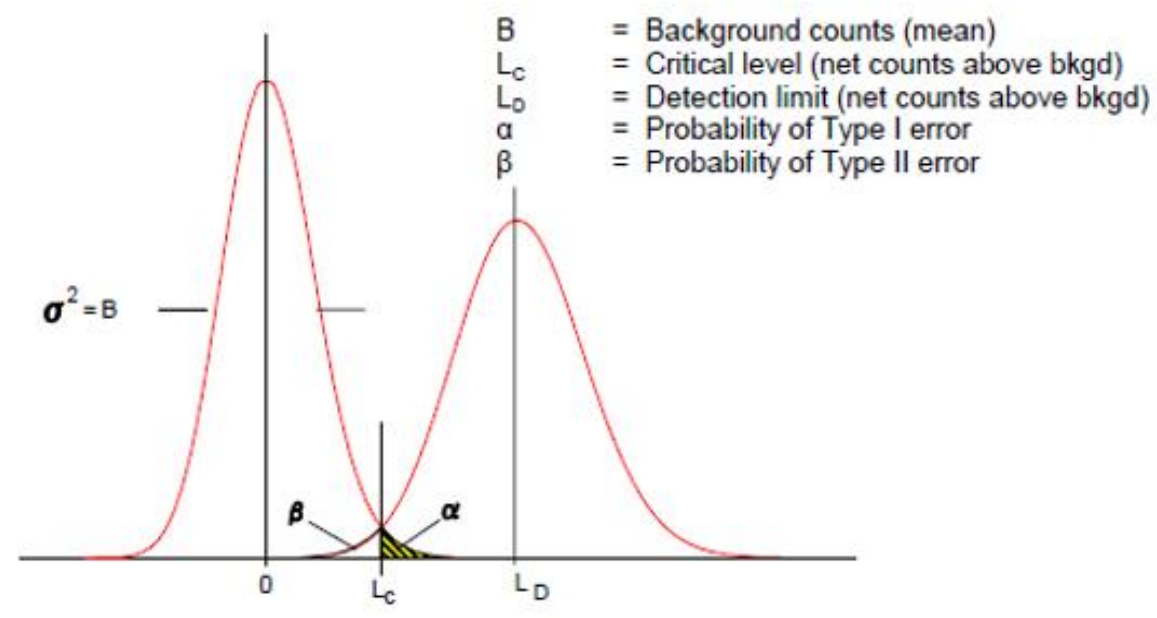

Figure 4 Critical Region and Detection Limit

where: $c_{B}$ are the counts of the blank, $T_{S}$ and $T_{B}$ are the counting live times of the sample and of the blank expressed in seconds; 1.645 is a constant that takes into account the statistical parameter $\alpha=5 \%$. The Critical Level indicates the minimum sample net counts ensuring that the probability of error $(\alpha)$ is below 5\% [24,25]. The detection sensitivity of the measurement system is defined by the Detection Limit $\left(L_{D}\right.$, see Eq. 4$)$, which indicates the minimum sample net counts that entails a probability error $(\beta)$ below $5 \%$.

$$
L_{D}[c p s]=\frac{3.29}{T_{S}} \cdot \sqrt{c_{B} \cdot\left(1+\frac{T_{S}}{T_{B}}\right)}
$$

Eq. 4

where: $c_{B}$ are the counts of the blank, $T_{S}$ and $T_{B}$ are the counting live times of the sample and of the blank expressed in seconds; 3.29 is a constant that takes into account the statistical parameters $(\alpha=5 \%$ and $\beta=5 \%)$ imposed by the comparison test.

Finally, the Minimum Detectable Activity concentration (MDAc, see Eq. 5) is used in the sample single hypothesis testing to verify if the measured activity concentration offers the appropriate level of statistical confidence with respect to the background. The statistical test is passed only if the measured activity concentration is greater than the MDAc, otherwise the measured datum is not acceptable.

$$
\operatorname{MDAc}\left[\frac{B q}{g}\right]=\frac{L_{D}}{\varepsilon \cdot m \cdot p_{i}}
$$

Eq. 5

where: $L_{D}$ is the Detection Limit, $\varepsilon$ is the efficiency and $m$ is the mass of the sample. In the case of the ${ }^{90} \mathrm{Y}$ MDAc evaluation, $p_{i}$ is the product of radiochemical specific parameters $\left(D_{Y}, Y_{\mathrm{Y}}\right.$ and $\left.Y_{\mathrm{Sr}}\right)$, while for the gamma emitting radionuclides $p_{i}$ is the $\mathrm{BR}$. 


\section{Results and discussion}

The results of the topsoil radiological survey preliminary to decommissioning are herein presented in detail. Thanks to the exhaustive bibliographic research in the literature and in the standardized methods and to the acquisition of the information about present and past activities of the nuclear facility, an accurate and reliable characterization was performed. The above described procedures were systematically employed to plan and perform the sampling campaign and to manipulate the samples before the radiochemical treatments and the radiometric measures. For each radionuclide considered in this study, the average of the MDAc calculated in all samples are reported in Table 2 along with their standard deviations.

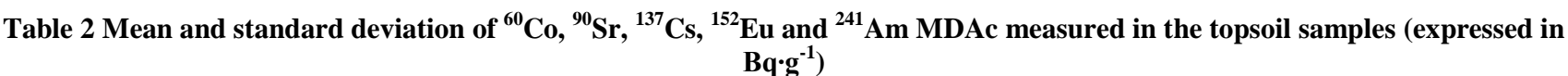

\begin{tabular}{cccccc}
\hline Nuclides & ${ }^{60} \mathbf{C o}$ & ${ }^{90} \mathbf{S r}$ & ${ }^{137} \mathbf{C s}$ & ${ }^{152} \mathbf{E u}$ & ${ }^{241} \mathbf{A m}$ \\
\hline Mean MDAc & $6.57 \cdot 10^{-4}$ & $1.80 \cdot 10^{-2}$ & $7.07 \cdot 10^{-4}$ & $1.62 \cdot 10^{-3}$ & $6.87 \cdot 10^{-3}$ \\
\hline $\begin{array}{c}\text { Standard deviation } \\
\text { MDAc }\end{array}$ & $1.54 \cdot 10^{-5}$ & $3.84 \cdot 10^{-3}$ & $1.36 \cdot 10^{-5}$ & $9.73 \cdot 10^{-6}$ & $1.35 \cdot 10^{-4}$ \\
\hline
\end{tabular}

In all the topsoil samples, both in the Non-Impacted zone and in the Impacted zone, the measured ${ }^{60} \mathrm{Co},{ }^{90} \mathrm{Sr}$, ${ }^{152} \mathrm{Eu}$ and ${ }^{241} \mathrm{Am}$ activity concentration values are below the respective MDAc, thus they are not distinguishable from the levels of the reference blank point. Concerning ${ }^{90} \mathrm{Sr}$, even if the obtained MDAc is considerably below the CL, it could be worthy reducing the MDAc by increasing the mass of the soil sample. To this end, an attempt was performed with the sample labelled " $1 \mathrm{R}$ ". The soil mass was increased of approximately 8 times (from $0.5 \mathrm{~g}$ of the standard procedure to about $4.2 \mathrm{~g}$ ), while the sample was treated following the same microwave-assisted digestion and radiochemical separation procedures. In this way, as it could be expected, the MDAc decreased from $1.26 \cdot 10^{-2} \mathrm{~Bq} \cdot \mathrm{g}^{-1}$ of the standard procedure to $1.98 \cdot 10^{-3} \mathrm{~Bq} \cdot \mathrm{g}^{-1}$. This modification to the procedure turned out to be promising, consequently attempts will be done to further increase the sample mass.

Conversely, the measured ${ }^{137} \mathrm{Cs}$ activity concentrations are orders of magnitude higher than those of the MDAc, consequently valid results can be derived from the analyses. The results are summarized in Figure 5, where: the red continuous line is the CL as defined by the EC RP 122 [9] and the green dotted line is the average regional value [16]. In order to display all the results in a single graphic, the sampling points are organized in macro areas according to their geographical distribution and to HSA. In particular, the Non-Impacted zone includes the 5 points collected outside the reactor surroundings and the Impacted zone is divided in four areas: West side (samples from \#1 to \#9, see the map of Figure 2), Reactor area (from \#10 to \#18), North-East side (from \#19 to \#23), South-East side (from \#24 to \#31). The activity concentration values of each macro area, as observable in Figure 5, are comparable with the average regional value $\left(7.6 \cdot 10^{-2} \mathrm{~Bq} \cdot \mathrm{g}^{-1}\right)$, that is mainly due to the Cernobyl accident of 1986 [16]. Furthermore, they are remarkably below the CL. It could be observed that in the topsoil samples collected in proximity of the buildings (i.e. the samples around the reactor and 19C buildings belonging to Reactor and North-East side areas respectively) the measured ${ }^{137} \mathrm{Cs}$ activity concentration values are slightly higher than the regional average level. This is probably due to the well-known rainwater accumulation phenomenon enhanced by nearby waterproof surfaces (in this case the surface consists in the gutter pipes of the building roofs) [26]. In fact, for several years both atmosphere and rainwater were affected by the radioactive contamination of the Černobyl fallout and of the nuclear weapon tests [27]. 


\section{Cs-137}

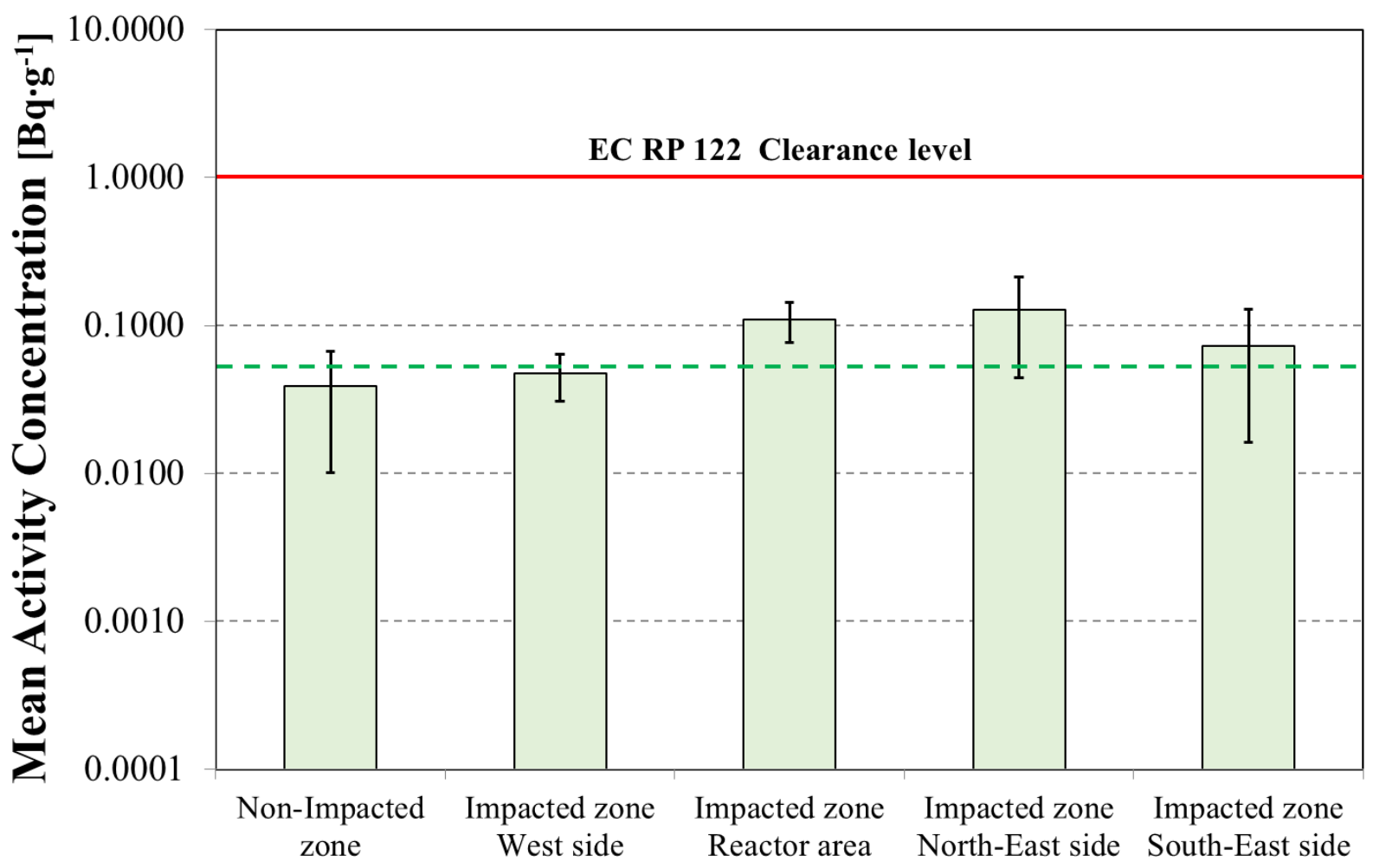

Figure $5^{137} \mathrm{Cs}$ mean activity concentration in $\mathrm{Bq} \cdot \mathrm{g}^{-1}$ as a function of the sampling area. The red continuous line is the $\mathrm{CL}$ as defined by the EC RP 122 [9] and the green dotted line is the average regional value [16].

This radiological pre-characterization showed that no contamination was spread outside the reactor building. With the exception of the well-known rainwater accumulation phenomenon [26], the activity concentration values of ${ }^{137} \mathrm{Cs}$ are orders of magnitude below the correspondent CL and not clearly discernible from the regional background $[9,16]$. Concerning ${ }^{90} \mathrm{Sr}$, the relatively high MDAc of the applied procedure prevented from obtaining valid results for that radionuclide. Nevertheless, since the MDAc is two orders of magnitude lower than the CL reported in RP 122, the levels of activity concentration are certainly admissible. Similarly for ${ }^{60} \mathrm{Co}$, ${ }^{152} \mathrm{Eu}$ and ${ }^{241} \mathrm{Am}$, the measured activity concentration values are by far lower than the correspondent MDAc and, consequently, of the CL.

\section{Conclusions}

This radiological survey was aimed at uncovering the radiological inventory of the topsoil surrounding the L$54 \mathrm{M}$ plant in order to obtain the radiological reference blank point before the decommissioning phase as well as assessing the possible contamination status of the site. With these aims, it was necessary to acquire all the available information on present and past activities of that nuclear facility before planning and performing the radiological analysis of the reactor surroundings. In order to guarantee an accurate and reliable characterization, an exhaustive bibliographic research in the literature and in the standardized methods was carried out. The results show that no contamination was spread outside the L-54M reactor building. In fact, in all topsoil samples 
the activity concentration values of the analyzed radionuclides are comparable with those of the regional background.

A different radiochemical procedure for the ${ }^{90} \mathrm{Sr}$ analyses will be pursued on randomly selected samples with the aim of increasing the treated mass and reducing the MDAc. Furthermore, with a view to ascertain whether contamination was accumulated by plants, several vegetation samples (e.g. moss, grass and cortex) will be collected, both in the Impacted and Non-Impacted zones. Finally, in order to acquire a deeper knowledge about the radionuclide inventory in the Impacted zone, the ${ }^{137} \mathrm{Cs}$ and ${ }^{90} \mathrm{Sr}$ activity concentration gradients will be evaluated up to $1 \mathrm{~m}$ depth in randomly selected points following preliminary hydrogeological site assessment [16]. In fact, the assessment of the radionuclide distribution could offer useful information on the radioactive contaminants migration in the soil as well as an interesting comparison with the results already obtained by ARPA (Regional Agency for the Environment Protection, in Italian: Agenzia Regionale per la Protezione dell'Ambiente) in another area of Milano classified as Non-Impacted zone [28].

\section{References}

[1] IAEA, Status of the Decommissioning of Nuclear Facilities around the World, Pub No. 201, Vienna, 2004

[2] OECD-NEA, Costs of Decommissioning Nuclear Power Plants, Pub No. 7201, 2016

[3] Sergio Terrani, Reactor and nuclear facilities of the CeSNEF site: Safety report (Il reattore e gli impianti nucleari del CeSNEF: Rapporto di Sicurezza), Technical Document written in Italian, Politecnico di Milano, 1961

[4] IAEA, Predisposal Management of Radioactive Waste Including Decommissioning, Safety Standards Series No. WS-R-2, IAEA, Vienna, 1999

[5] IAEA, Decommissioning of Nuclear Power Plants and Research Reactors, Safety Standards Series No. WSG-2.1, Vienna, 1999

[6] IAEA, Advancing Implementation of Decommissioning and Environmental Remediation Programmes, Nuclear Energy Series No. NW-T-1.10, Vienna, 2016

[7] NRC, EPA, DoE, DoD, Multi-Agency Radiation Survey and Site Investigation Manual (MARSSIM), 2000

[8] CND (FP6), Environmental Radiation Survey and Site Execution Manual (EURSSEM), 2010

[9] EC, RP 122 - Recommendation on Practical use of concepts of clearance and exemption: Part I, 2000

[10] D. M. 4/8/2000 - Caorso Nuclear Power Plant, Decommissioning according to Articles No. 55 and 56 of the 230/95 Legislative Decree (Centrale Nucleare di Caorso, disattivazione ai sensi degli artt. 55 e 56 nel D. Lgs 230/95), Document written in Italian, 2000

[11] Argonne National Laboratory - Biological and Medical Division, Annual Report 1964, Argonne, 1964

[12] Codispoti L., Radiological pre-characterization of site and structures of the L-54M CeSNEF plant (Precaratterizzazione radiologica del sito e delle strutture dell'impianto L-54M CeSNEF) Master thesis written in Italian, Politecnico di Milano, 2015

[13] ISO 11932 - Activity measurements of solid materials considered for recycling, re-use or disposal as nonradioactive waste, 1996

[14] ISO 18589-2: Measurement of radioactivity in the environment - Soil - Guidance for the selection of the sampling strategy, sampling and pre-treatment of samples, 2007 
[15] ASTM C999: Standard practice for soil sample preparation for the determination of radionuclides, 2010

[16] Forte M., Rusconi R., Tolomei S., Sgorbati G., ${ }^{137} \mathrm{Cs}$ and ${ }^{90} \mathrm{Sr}$ concentration in Lombardia soils: Measurements and migration profile analysis. European IRPA Congress 2002, Firenze, 2002

[17] ISO 18589-3: Measurement of radioactivity in the environment - Soil - Measurement of gamma-emitting radionuclides, 2007

[18] ASTM C1402: Standard guide for high-resolution gamma-ray spectrometry of soil samples, 2009

[19] ISO 18589-5: Measurement of radioactivity in the environment - Soil - Measurement of strontium 90, 2009

[20] Eichrom Technologies, Inc., Strontium 89,90 in Water (SRW01), Analytical Procedures, 2003

[21] EPA 3052: Microwave assisted acid digestion of siliceous and organically based matrices, 1996

[22] Ross H., Theory and application of Cerenkov effect, Oak Ridge National Laboratory - Analytical Chemistry Division, Oak Ridge, Tennessee, USA, 1976

[23] Ross H., Measurement of $\beta$ emitting nuclides using Cerenkov radiation. Anal. Chem., 41:1260-1265, 1969

[24] ISO 11929-1: Determination of the characteristic limits (decision threshold, detection limit and limits of the confidence interval) for measurements of ionizing radiation - Fundamentals and application, 2010

[25] So.g.i.n., Determination of ${ }^{90} \mathrm{Sr}$ (Determinazione dello ${ }^{90} \mathrm{Sr}$ ), Document written in Italian, PP-04, Rev. No. 1, 1998

[26] Holgye Z., Schlesingerová E., Tecl J., Filgas R., ${ }^{238} \mathrm{Pu},{ }^{239,240} \mathrm{Pu},{ }^{241} \mathrm{Am},{ }^{90} \mathrm{Sr}$ and ${ }^{137} \mathrm{Cs}$ in soils around nuclear research centre Rez, near Prague. J. Environ. Radioactiv., 71:115-125, 2004

[27] Al Hamarneh I., Wreikat A., Toukan K., Radioactivity concentrations of ${ }^{40} \mathrm{~K},{ }^{134} \mathrm{Cs},{ }^{137} \mathrm{Cs},{ }^{90} \mathrm{Sr},{ }^{241} \mathrm{Am},{ }^{238} \mathrm{Pu}$ and ${ }^{239+240} \mathrm{Pu}$ radionuclides in Jordanian soil samples. J. Environ. Radioactiv., 67:53-67, 2003

[28] ARPA Lombardia, Manual for large scale radiological emergency (Manuale per le emergenze radiologiche su vasca scala), Document written in Italian, cod: IO AF 010, Milano, 2015 